\title{
Could CRISPR be the solution for gene editing's Gordian knot?
}

\author{
Hao Fang • William Wang
}

Received: 15 July 2016/Accepted: 1 September 2016/Published online: 10 September 2016

C) Springer Science+Business Media Dordrecht 2016

Clustered regularly interspaced short palindromic repeats (CRISPR)/Cas (CRISPR associated) system has received an explosive amount of interest in scientific world since 2013. The system, composed of Cas9, subgenomic RNA (sgRNA) from crRNA, and CRISPR RNA, functions through base pairing DNA double-strand break (DSB) initiation-induced homology-directed repair (HDR) or non-homologous end joining (NHEJ). The three main types are represented by a specific Cas protein, type I by Cas3, type II by Cas 9 , and type III by Cas10, which still need to be further explored. Reprograming Cas9 into catalytically dead Cas9 (dCas9) results in site-specific DNA-binding domain which can transport desired domains to the target locus effectively activating (CRISPRa) or repressing (CRISPRi) transcription due to its reversibility. CRISPR/ Cas9 system could lead to significant advances in epigenetics, cell functions, and gene therapy due to its cost, accuracy, and time efficiency which were major obstacles previously for genome editing. With a potential increase in CRISPR/Cas9-based tool usage, an emphasis must be put on detecting possible unwanted or unexpected side effects together with discussions regarding its applications in therapeutics for inherited diseases, cancer, and infectious disease eradication.

H. Fang $(\bowtie)$

Department of Anaesthesiology, Jinshan Hospital, Fudan University, 1508 Longhang Road, Shanghai 201508, China e-mail: drfanghao@163.com

W. Wang

School of Dentistry, University of Plymouth, Plymouth, UK
Zinc-finger nucleases (ZFNs), transcription activatorlike effector nucleases (TALENs), and CRISPR/Cas9 are the three prominent methods used in human-induced pluripotent stem cells (hiPSC) since genetic modifications can be efficiently localized at the generated DSB. Currently, ZFN is being phased out due to its high technical skill requirement and inefficiency. TALEN is more efficient than ZFN and requires $\sim 1800$ bp for a target site, whereas CRISPR/Cas9 only requires $20 \mathrm{bp}$, increasing both screening targets and efficiency. However, TALEN has less restrictions in genetic site targeting as the presence of thymine at each $5^{\prime}$ end is sufficient compared to CRISPR/Cas9 which requires a protospacer adjacent motif (PAM) sequence. Additionally, due to the presence of the Fokl nuclease in TALEN, it produces fewer off-target effects compared to current CRISPR/Cas9-based tools. Studies have demonstrated that CRISPR/Cas9 could successfully knock out cell lines resulting in faster generation of iPSC cell lines with specific genes (Chen et al. 2015) while another study attempted to reprogram HEK293T cells into iPSC through activating human OCT4 promoter through dCas9-VP64 (Hu et al. 2014). CRISPR/Cas9 can be utilized in gene correction in patient-specific iPSCs for muscular dystrophy and haemophilia to improve cell enrichment, drug screening, and differentiation protocols and simplify in vitro assays due to disease-specific single nucleotide mutations. Limitations such as non-existing biological readouts for the impact on cells and possible offtarget activities remain an issue; however, CRISPR/Cas9 has made progress in the safety of designer nucleases which is one of the main concerns regarding gene editing in iPSCs. CRISPR/Cas9 coupled with iPSC technology 
enables cell materials to be generated from patients which could be directly correlated with the clinical phenotype, though this remains a challenge as iPSC clones from a donor vary in differentiation capacity and cultural behaviour (Merkert and Martin 2016).

CRISPR/Cas9 has a potential to be used as a gene drive to modify genes that are undesirable in species which transmit infectious diseases. For example, gene drives within mosquitoes could be targeted to block malaria transmission, an example being Anopheles stephensi which were adapted from mutagenic chain reaction (Gantz et al. 2015). Infectious diseases spread by insects have decreased throughout the years, but mortality rates still remain high with 438,000 deaths caused by malaria alone in 2015 (WHO 2015). Invasive species that negatively impact local ecosystems could also be regulated and controlled as many of the current solutions are either inefficient or have an adverse impact on natural species within an ecosystem; CRISPR/Cas9 would enable the specific targeting of certain species that are invasive without causing harm to other inhabitants. These possibilities are reduced and influenced by possible limitations such as the required presence of the PAM sequence and a time scale of generations to manifest. Short-spanned creature such as insects and rats would be achievable whereas long-lived animals could require an alternative solution. This also assumes that the trait will aid in organism survival; if it has a detrimental trait, there is the possibility that it would be phased out by natural selection.

Safety measurements should be considered, e.g. a "reversal drive" which could overwrite the initial gene drive and remove unwanted off effects (Esvelt et al. 2014). Damage caused during the implementation of the "reversal drive" would be irreversible emphasizing the importance of a swift and rapid response period. We should question whether it would be effective in longer life-spanned species due to varying factors. There is an urgent need of evidence-based studies using an engineered endonuclease gene drive that could be successfully distributed within a wild population. Ethical issues on nonnatural involvement in nature's development should also be considered, before any clinical application be taken. CRISPR/Cas9 is a promising method to combat infectious diseases spread through insects, although challenges on its limitations and effectiveness still remain.

Cas9 results in irreversible effects and a higher possibility for off-target effects compared to dCas9 which decreases undesired and reversible effects to off-targets in regard to two distinct sgRNA sequences. Off-target effects and incomplete knockdowns of RNA interference (RNAi) methods on genome wide screens have been a prevalent issue. CRISPRi achieved a high knockdown rate with few off-target effects, and although off-targets were similar to TALE and other RNAi methods, it has potential to be further optimized (Gilbert et al. 2013). The oligonucleotide synthesis makes sgRNAs in Cas9 easier to be designed and cloned to achieve genome-wide screens. The function of dCas9-KRAB/sgRNA could modulate gene expression and generate higher sensitivity than shRNA to identify resistance-associated genes to ricin toxin in human cells (Gilbert et al. 2014). CRISPR/dCas9 could also be modified to screen for function gains and to increase the scope of RNAi regulation in cells. Transcription repression will vary between genes in mammalian cells, and the binding of dCas9-sgRNA complex could be hindered by endogenous chromatin.

A transcriptional start site (TSS) was annotated using FANTOM5/GAGE promoter atlas and demonstrated that CRISPRi efficiency increased once the correct TSS was identified (Radzisheuskaya et al. 2016). Preferences of sgRNA were considered and emphasized on chromatin accessibility of the target site, providing a possible guideline for a more efficient CRISPRi assay design. Metabolic engineering techniques based on dCas9 have been applied to unicellular organisms, with a potential for application in a complex multicellular organism. It would be exciting to implement dCas9 in biosensing and bioremediation through metabolic networks, resulting in desired traits to cells such as stress resistance. The applications of dCas9 may be extended to image genomic loci in cells, identify crucial proteins involved in pluripotency or differentiation, or control bacterial population through bacterial conjugation.

We should explore more potentials of Cas 9 in clinical therapies. Cas 9 can change the sensitivity and responses of cells to discovered drug, evidenced by the finding that CRISPR/Cas RGNs could alter the resistance of multidrugs in the model of a Galleria mellonella infection (Citorik et al. 2014). The preliminary findings from Church and his colleagues showed that Cas9 could inactivate 62 porcine endogenous retroviruses (PERVs) in pig embryos (Nature News 2016). Such discovery could result in the first suitable non-human organ donor and have a significant impact in tissue engineering and regenerative medicine, as exampled to exhibit benefit from CRISPR/Cas9. In addition, the use of CRISPR as a screen for cancer genes has already been 
employed to identify and confirm potential genes with therapeutic potential. CRISPR can be used to edit selected targeted genes and generate mutation-specific or dominant cancerous cell lines to narrow down targeted genes and pathways in oncogenesis. CRISPR also has great potential for screening of drug efficacy and toxicity. For example, CRISPR-edited cancer cells with a specific gene mutation can be applied to define the sensitivity, response, and resistance of CRISPR-edited cells to the drug. The alterations of CRISPR-edited cell function by drug will confirm selected targets of potential treatments prior to or during clinical application. This will be a new future for clinical validation and screening of drugs and a new potential for clinical application of CRISPR to measure possible effects and toxicity profiles of drugs to mutation-specific cells.

CRISPR/Cas9 has a great future as an efficient method in gene engineering, although a number of drawbacks and limitations have to be overcome. CRISPR/Cas9modified expression and function of epidermal growth factors or targeting V-Ki-ras2 Kirsten rat sarcoma viral oncogene homologue (KRAS) or histone deacetylase can be also considered for therapy in oncology and inheritable diseases. One of the most important issues is to address ethical dilemmas prior to implementation. With the discovery and development of gene therapy products, CRISPR/Cas9-based tools become more and more critical and necessary in clinical applications. We should carefully examine known and unknown limitations and how CRISPR can be further optimized and address ethical concerns in its application. Effective communication between the scientific community, industries, authorities, and public will become a necessity to determine the best use. It is crucial to remember the importance of transparency for the public throughout the process of discovery, development, and application of the technology.

\section{References}

Chen Y, Cao J, Xiong M, et al. Engineering human stem cell lines with inducible gene knockout using CRISPR/Cas9. Cell Stem Cell. 2015;17(2):233-44.

Citorik RJ, Mimee M, Lu TK. Sequence-specific antimicrobials using efficiently delivered RNA-guided nucleases. Nat Biotechnol. 2014;32:1141-5.

Esvelt KM, Smidler AL, Catteruccia F, Church GM. Concerning RNA-guided gene drives for the alteration of wild populations. Elife. 2014; 03401.

Gantz VM, Jasinskiene N, Tatarenkova O, et al. Highly efficient Cas9-mediated gene drive for population modification of the malaria vector mosquito Anophelesstephensi. Proc Natl Acad Sci U S A. 2015;112(49):E6736-43.

Gilbert LA, Larson MH, Morsut L, Liu Z, Brar GA, Torres SE, Stern-Ginossar N, Brandman O, Whitehead EH, Doudna JA, Lim WA, Weissman JS, Qi LS. CRISPR-mediated modular RNA-guided regulation of transcription in eukaryotes. Cell. 2013;154:442-51.

Gilbert LA, Horlbeck MA, Adamson B, Villalta JE, Chen Y, Whitehead EH, Guimaraes C, Panning B, Ploegh HL, Bassik MC, Qi LS, Kampmann M, Weissman JS. Genomescale CRISPR-mediated control of Gene repression and activation. Cell. 2014;159:647-61.

Hu J, Lei Y, Wong WK, et al. Direct activation of human and mouse Oct4 genes using engineered TALE and Cas9 transcription factors. Nucleic Acids Res. 2014;42:437590.

Merkert S \& Martin U. Site-specific genome engineering in human pluripotent stem cells. Int J Mol Sci. 2016;17(7):E1000.

Nature News \& Comment: Gene-editing record smashed in pigs. Nature News. [ONLINE]. Available at: http://www.nature. $\mathrm{com} /$ news/gene-editing-record-smashed-in-pigs-1.18525 (2016).

Radzisheuskaya A, Shlyueva D, Muller I \& Helin K. Optimizing sgRNA position markedly improves the efficiency of CRISPR/dCas9-mediated transcriptional repression. Nucleic Acids Res. 2016. pii: gkw583.

World Health Organization: World Malaria Report 2015. Geneva, [ONLINE]. Available at: http://www.who.int/malaria /publications/world-malaria-report-2015/report/en/ (2015). 